\title{
Sevoflurane postconditioning ameliorates neuronal migration disorder through Reelin/Dab1 and improves long-term cognition in neonatal rats after hypoxic-ischemic injury
}

\author{
Yahan Zhang \\ Shengjing Hospital of China Medical University \\ Qiushi Gao \\ Shengjing Hospital of China Medical University \\ Ziyi Wu \\ Shengjing Hospital of China Medical University \\ Hang Xue \\ Shengjing Hospital of China Medical University \\ Chang Li \\ Shengjing Hospital of China Medical University \\ Ping Zhao ( $\nabla$ zhaoping_sj@163.com ) \\ https://orcid.org/0000-0001-6392-5391
}

\section{Research}

Keywords: Sevoflurane postconditioning, Hippocampal Dentate gyrus, hypoxia-ischemia, Reelin, longterm neurocognition

Posted Date: February 24th, 2020

DOI: https://doi.org/10.21203/rs.2.24336/v1

License: (c) (i) This work is licensed under a Creative Commons Attribution 4.0 International License. Read Full License

Version of Record: A version of this preprint was published at Neurotoxicity Research on July 5th, 2021. See the published version at https://doi.org/10.1007/s12640-021-00377-3. 


\section{Abstract}

Sevoflurane postconditioning (SPC) had been reported to attenuate developing brain injury after hypoxiaischemia encephalopathy (HIE)via inhibiting neural necrosis and autophagy process. Moreover, recent report elucidated sevoflurane may involve in neural cells migration after injury. Here we hypothesize neuronal migration and long-term cognition were ruined after HIE and SPC alleviated these injuries .Classical Rice-Vannucci model of Hypoxia-ischemia was conducted on P7 pups, which was followed by SPC at the 1 minimum alveolar concentration (MAC 2.4\%) for 30 min. Piceatannol which can cleave Reelin into proteolytic fragments was used to detect whether Reelin/Dab1 is involved in neuroprotection exerted by SPC. Our findings suggest that hypoxia-ischemia disrupted cytoarchitecture of dentate gyrus (DG) by inhibiting the migration of dentate neurons of hippocampus, which may eventually lead to longterm cognition deficits. However, SPC could relieve the restricted hippocampal neurons from the subgranular zone of hippocampi combined with the repair of hippocampal-dependent memory function damaged by HIE through attenuating the overactivation of the Reelin/Dab1pathway. Taken together, these results demonstrate that SPC plays a pivotal role in ameliorating neuronal migration disorder and maintain normal cytoarchitecture and spatial learning ability of DG by regulating the Reelin/Dab1 downstream signaling pathway. This indicates the potential therapeutic use of SPC in treating HIE perinatally.

\section{Introduction}

Neonatal hypoxic-ischemic encephalopathy (HIE) is one of the prevalent causes of severe neurological morbidity in neonates, which may lead to devasting neurological, cognitive, and behavioral disorders. The incidence of HIE is approximately $1 / 1000$ in live births, and the survivors may still suffer from severe sequelae ${ }^{1,2}$. To date, in addition to hypothermia, very few clinically promising therapies have been reported. However, the long-term efficacy of these therapies in terms of alleviating brain damage after HIE is still very low.

The development of hippocampal neurons is a sensitive, multistep process involving progenitor proliferation from the neurogenesis niche dentate gyrus (DG ) zone at a low rate, migration to the final location, and differentiation and synaptogenesis until integration with the existing neuronal circuit ${ }^{34}$. However, although the central nervous system initiates neural self-repairment after injury, including reactive neurogenesis, it is still unable to repair severe injuries ${ }^{5}$. Previous evidence has also confirmed that precise neuronal migration is vital for the establishment of hippocampal functional neuronal circuits. There are more than 9,000 immature neurons being sent daily from the subgranular zone (SGZ) of DG, and this process continues throughout the life, with the migration of neurons from the subgranular layer outward to the granular and multiple molecular layers. Disruption of this process can lead to neurological disorders such as schizophrenia ${ }^{6}$. Therefore, a medicine that can promote effective neural migration from DG after hypoxic-ischemic injury may be a promising therapy. 
Sevoflurane is a widely used inhalational anesthetic in pediatric clinics. Mounting evidence support the idea that sevoflurane postconditioning (SPC) may alleviate neurological deficits by inhibiting neural apoptosis, suppressing overactive autophagy process, and inhibiting inflammation ${ }^{6,7}$. Recent evidence proved that sevoflurane promoted the migration of microglia and astrocytes toward ischemic foci to repair the injury after transient ischemic injury ${ }^{8}$; this indicated that sevoflurane may exert its neuroprotective effect by aligning hippocampal neural cell orientation 9,10 . Yet, it remains unclear whether SPC affects the hippocampal DG reconstruction process after HIE and the underlying mechanism of its action.

Reelin is a large extracellular molecular protein secreted by Cajal-Retzius cells, which plays a key role in the correct positioning of cortical and hippocampal neurons ${ }^{11}$. It consists of eight Reelin repeats, and its $\mathrm{N}$-terminal region is required for signal transduction ${ }^{12,13}$. The research on Reelin mutant mice, called "reeler" phenotype, has contributed to our knowledge of the Reelin effect on orchestrating the arrangement of neurons in the cortical layer, hippocampal layer, and other regions such as cerebellum ${ }^{14}$.

Studies on canonical and noncanonical Reelin signaling pathways have shown that Reelin can influence the neuron migration process through multiple ways, including binding to the apolipoprotein E receptor 2 or the very low-density lipoprotein receptor, which subsequently activates intracellular Dab1 or N-cadherin to participate in neuron migration, synaptic plasticity modulation, and neurotransmitter release process ${ }^{15}$. Previous investigations have revealed that maternal hypoxia had a direct influence on hippocampal neuronal migration by altering Reelin expression and ultimately leading to hippocampal lamination malformation 16,17 .

On the basis of previous evidence, in the present study, we aimed to test the hypothesis that hypoxic and ischemic stress can induce neuron arrangement disorders in DG neurons of the hippocampus and thereby cause long-term neurological deficits. SPC may alleviate these neurological impairments by regulating Reelin expression and the downstream Reelin/Dab1 signaling pathway and facilitate long-term spatial learning and cognition in neonatal rats after hypoxic-ischemic injury.

\section{Materials And Methods}

Experimental animals and grouping

Postnatal day 7 (PND7) Sprague-Dawley rat pups were used in this study because of peak neurogenesis of the hippocampus. Housing conditions of the rats complied with the regulations of the National Animal Experiment Center. Briefly, the pups were housed in a room at $25 \pm 2 \circ \mathrm{C}$ under a 12 -h light/dark cycle, with free access to adequate food and water. All experimental procedures were approved by The Laboratory Animal Care Committee of China Medical University (Shenyang, China; Approval No.: 2016PS337K) and conformed to National Institutes of Health Guidelines for the Care and Use of Laboratory Animals.

Grouping 
Pups of both sexes were randomly divided into four groups: Sham group (Sham), HIE group (HI), HIE with Sevoflurane postconditioning group $(\mathrm{HI}+\mathrm{Sev}$,$) , and \mathrm{HIE}$ with Sevoflurane postconditioning + Piceatannol drug group $(\mathrm{HI}+\mathrm{Sev}+\mathrm{G})$

Neonatal HIE model and drug administration

Neonatal HIE model protocol was based on a previously described protocol ${ }^{18}$. Briefly, sevofluraneanesthetized pups (12-20 g body weight) were operated for permanent ligation of the left common carotid artery. After recovering for $2 \mathrm{~h}$, the pups were ventilated with $30 \% 02$ and $70 \% \mathrm{~N}$ at the flow rate of $2 \mathrm{~L} / \mathrm{min}$ for $2 \mathrm{~h}$ in the Sham group and $8 \% \mathrm{O} 2$ and 92\% N in the HIE groups. SPC at 1 minimum alveolar concentration (MAC 2.4\%) was conducted immediately for 30 min after asphyxia by using a gas monitoring device (Datex-Ohmeda Inc., Tewksbury, MA, USA).

Drug administration in vivo

Piceatannol ( $30 \mu \mathrm{M}$, Sigma-Aldrich, USA), a highly selective inhibitor of ADAMTS-4 and ADAMTS-5 that specifically cleaves the N-terminal region (N-R2) of Reelin into proteolytic fragments, was injected into the left lateral ventricle of the pups by using a $5-\mu \mathrm{L}$ Hamilton syringe 30 min before hypoxia in the $\mathrm{HI}+\mathrm{Sev}+$ $\mathrm{G}$ group, while the HI group received an equal volume of DMSO.

Pulse labeling study by bromodeoxyuridine injection

5-Bromo-2-deoxyuridine ( $\mathrm{BrdU}$ ) pulse birth-labeling method for detecting neuron migration has been considered as a potential and accepted method $16,19,20$. To determine the migration of newborn neurons, BrdU (B5002, Sigma-Aldrich) was intraperitoneally injected into dams at the dose of $150 \mathrm{mg} / \mathrm{kg}$ per day for 3 days consecutively from PND6 to PND9. The pups were sacrificed, and their brains were harvested on PND14 and PND21.

Tissue processing and immunofluorescence staining

For the immunofluorescence assay, the pups were deeply anesthetized with pentobarbital $(80 \mathrm{mg} / \mathrm{kg})$, and their brains were harvested. Coronal sections of paraffin-fixed brain were cut consecutively at the thickness of 3-3.5 $\mu \mathrm{m}$. Similar to that for BrdU staining, the sections were treated with $2 \mathrm{~N} \mathrm{HCl}$ at $37^{\circ} \mathrm{C}$ for $30 \mathrm{~min}$ for denaturing DNA, followed by neutralization with $0.1 \mathrm{M}$ borate buffer ( $\mathrm{pH} 8.5)$. The sections were then blocked with $5 \%$ fetal bovine serum for $30 \mathrm{~min}$ and then incubated overnight with primary antibodies. On the next day, the slices were incubated with one or two types of secondary antibodies for single or double staining for $4 \mathrm{~h}$ at room temperature, and cell nuclei were counterstained with DAPI. The following antibodies were used: anti-mouse Reelin (1:200; G10 Abcam, Cambridge, UK), anti-rabbit BrdU (1:100; Abcam), and anti-mouse Neun (1:100; Cell Signaling Technology, Boston, MA, USA). Cell nuclei were labeled with DAPI and then coverslipped with an antifade reagent (P0128S, Beyotime, China). The ratio of Reelin/Dab1-coexpressing cells to the total Reelin-positive cells was calculated for evaluating Reelin and Dab1 expression. To evaluate the number of Reelin-positive cells at PND7, PND8, and PND14, 
we counted the number of positive cells and then divided this number by the total cell number in a fixed area $(1,000 \times 1,000$ pixels) by using NIS-Elements AR Analysis 4.50 .00 software; this procedure was performed for every $50-\mu \mathrm{m}$ slice coronal sections, for a total of 5 sections per brain. To evaluate the combination of Reelin and Dab1 expression under different drug treatments, the ratio of double-positive cell number to Reelin-positive cell number was calculated. According to previous reports on neuron migration, we calculated the ratio of BrdU/Neun co-expressing cells to total BrdU-positive cells and compared the percentages in the subgranular cell layer (SGZ) and the granular cell layer (GCL) on PND14 and PND21. Representative images were photographed with a Nikon C1 microscope by a skilled pathologist who was blinded to the experimental conditions and groups.

Nissl staining

Nissl staining was performed on PND14 by using a staining kit. The coronal sections were stained according to the manufacturer's instructions (G1430, Solarbio, China). Neural orientation and distribution of the hippocampal neurons of the DG region were captured using a digital microscope camera. The relative ratio of neuronal density of DG was analyzed with Image J software.

Western blotting analysis

Hippocampal DG tissue was carefully dissected from the brains of the pups by using an anatomical microscope (Nikon SMZ445) at PND8 ( $n=5$ per group). Frozen hippocampi DG was cut and then lysed on ice for $30 \mathrm{~min}$. The lysate was centrifuged, and the total protein concentration was measured using the BCA Protein Assay Kit (P0010; Beyotime). The proteins were separated by electrophoresis on $8 \%$ or 10\% SDS-PAGE gel and then electrotransferred to polyvinylidene fluoride membranes (IPVH0010; Millipore, Germany). The target proteins were incubated with the specific primary and second antibodies. The following primary antibodies were used: GAPDH (1:1000; Cell Signaling Technology); Reelin (1:1000; G10 Abcam); Dab1 (1:500; Biorbyt, Cambridge, UK); Phospho-Dab1 (phospho-Tyr198, 1:100; Biorbyt); GSK-3 $\beta$ (1:1000; Cell Signaling Technology); Phospho-GSK-3 $\beta$ (Ser9, 1:500; Cell Signaling Technology); Tau (1:400; Sigma-Aldrich); and Phospho-tau ser396 (1:400; Abcam) The band intensity was quantified using Image PRO PLUS software.

\section{Ethological Tests}

Three behavioral tests were conducted on days PND28-P34 as previously described.

Open field test

To evaluate anxiety and locomotor behavior, the rats were placed in a $100 \mathrm{~cm}(W) \times 100 \mathrm{~cm}(D) \times 40 \mathrm{~cm}$ $(\mathrm{H})$ arena with opaque sidewalls equipped with infrared detectors for 10 min (Noldus Ethovision XT, Netherland), and the movement distance, time spent in the center and along the wall, movement velocity, and fecal pellets were recorded for anxiety mood evaluation.

Morris water maze test 
Spatial learning and memory ability of rats were tested by the Morris water maze (MWM) test from PND29 to PND34. Briefly, during the first consecutive 5 days, the rats were placed into a circular, opaque pool (1.6 m diameter, $60 \mathrm{~cm}$ height) for searching an invisible platform within $90 \mathrm{~s}$. Once failed, they were forced to stay on it for another $20 \mathrm{~s}$. During the spatial probe test, the rats were released from the opposite quadrant with the platform removed away and permitted to swim for $90 \mathrm{~s}$. The paths of each animal were recorded by infrared detectors, and the data were analyzed by an image analysis software (Noldus Ethovision XT)

\section{8-Arm radial maze test}

The 8-arm radial maze (RAM) test was conducted as described previously ${ }^{21}$. During the acclimatization phase, the rats were allowed to explore freely for $10 \mathrm{~min}$ with all arms baited after controlling their weight until $85-90 \%$ of free-feeding body weight was achieved. During the next 5 days of the training phase, four of the eight arms were baited randomly, and the rats were trained twice a day to find the food reward with an interval of $1 \mathrm{~h}$ between the trials. Each trial was terminated within $10 \mathrm{~min}$ or before $10 \mathrm{~min}$ if the rats found all food rewards. During the choice phase, all arms were unbaited, and the rats were allowed to find the food reward within $10 \mathrm{~min}$. The total travelled distance, reference memory error (RME), and working memory error (WME) were recorded and analyzed using an image analysis software (Noldus Ethovision XT).

Statistical analysis

Data are presented as mean \pm standard deviation (SD). All data were analyzed with SPSS 20.0 or GraphPad Prism 7.0. The data were analyzed using Bartlett's test for equal variances and Shapiro-Wilk test for normality. One-way analysis of variance (ANOVA) followed by Newman-Keuls post hoc test was used. Data of escape latency in MWM and the ratio of neuronal migration were analyzed using two-way ANOVA for repeated measurements. Differences with $p<0.05$ were considered to be statistically significant.

\section{Results}

Neuron migration process was perturbed and DG neuron distribution was disordered after HIE, and SPC alleviated these injuries

To better visualize neuron migration from the SGZ to the superficial GCL, the BrdU pulse labeling protocol was used (Supplementary Figure S1). Initially, the migration of the pulse-labeled cells to the destination was detected and the ratio of BrdU/neuron cells to the total BrdU-positive cells in each layer was calculated (Fig. 1A, PND14; 1B, PND21). The ratio of BrdU+/neuron + cells in the SGZ layer to BrdU + cells in the whole layer was enhanced in the HI group as compared to that in the Sham group on PND14 and PND21. This change in the ratio indicated that a large number of labeled neurons were restricted to the SGZ rather than migrating outward (Fig. 1C, D, HI versus Sham, $p<0.05$ and $p<0.05$ on PND14 and PND21, respectively). However, the ratio of BrdU+/neuron + cells in the GCL to the total BrdU + cells in the 
whole layer was decreased as compared to that in the Sham group (Fig. 1C,D, HI versus Sham, $\mathrm{p}<0.01$ and $p<0.01$ on PND14 and PND21, respectively). To exclude the interference of reactive neurogenesis in $\mathrm{BrdU}+$ cells after HIE, the total BrdU + cells were counted, and the data showed no significant difference among all groups (Supplementary Fig. 2). SPC decreased the elevated number of neuronal cells restricted in the SGZ zone and increased the ratio in the GCL (Fig. 1C, D, HI + Sev versus HI, p < 0.05, p< 0.05; Fig. 1C, $D, H I+$ Sev versus $H I, p<0.05, p<0.01$ on PND14 and PND21, respectively).

Nissl staining was conducted on PND14 to study the distribution of hippocampal DG neurons (Fig. 1E). As compared to the Sham group, the HI group showed decreased neuron ratio with rare cytoplasm and few Nissl bodies and disorganized neurons without polarity in DG (Fig. 1F, HI versus Sham, p < 0.001). SPC alleviated neuron loss and promoted reorganization of the neurons with proper orientation (Fig. 1F, $\mathrm{HI}+\mathrm{Sev}$ versus $\mathrm{HI}, \mathrm{p}<0.01$ ).

\section{Reelin expression was enhanced after HIE}

Here, we investigate the link between Reelin expression and SPC protective effect. Our data showed that rats in the HI group persistently expressed more Reelin-positive cells than those in the Sham group on PND8, PND14, and PND21 (Fig. 2A-F, HI versus Sham, $p<0.05, p<0.01$, and $p<0.01$ on PND8, PND14, and PND21, respectively). SPC significantly downregulated Reelin expression (Fig. 2A-F HI+ Sev versus $\mathrm{HI}, \mathrm{p}<0.05, \mathrm{p}<0.01$, and $\mathrm{p}<0.01$ on PND8, PND14, and PND21, respectively).

SPC shows protective effect by repairing the distribution of DG neurons and establishing neuronal migration by suppressing Reelin function.

Piceatannol, which effectively inhibits the activity of the Reelin proteolytic enzyme (ADAMTS-4/5), was used to test the protective effect of SPC on the HIE target Reelin. To verify the Reelin/Dab1 intercellular combined reactions that may activate the downstream intracellular reactions, immunofluorescence staining of co-expression of Reelin and Dab1 was conducted. The co-expression of Reelin and Dab1 was significantly enhanced after HIE, and SPC significantly inhibited the elevated expression (Fig. 3A-F, HI versus Sham, $p<0.01, p<0.001$, and $p<0.001 ; H I+$ Sev versus $H I, p<0.01, p<0.05$, and $p<0.01$ on PND8, PND14, and PND21, respectively). The beneficial effects of SPC were blocked by Piceatannol (Fig. 3A-F $\mathrm{HI}+\mathrm{Sev}+\mathrm{G}$ versus $\mathrm{HI}+\mathrm{Sev}, \mathrm{p}<0.01, \mathrm{p}<0.01, \mathrm{p}<0.05$ on PND8, PND14, and PND21, respectively)

Consistent with the above findings, there were more BrdU/Neun-labeled cells in the SGZ and GCL zones of rats of the HI group than those in rats of the Sham group (Fig. 4C, D, HI versus Sham, p<0.01, p $<0.05$ in SGZ; $p<0.05, p<0.01$ in GCL on PND14 and PND21, respectively). This finding indicated that a large amount of neurons were restricted to the basal zone of $D G$, and this high ratio was decreased by SPC (Fig. 4C, D, HI versus Sham, $p<0.05, p<0.05$ in SGZ; $p<0.01, p<0.01$ in GCL on PND14 and PND21, respectively). Piceatannol blocked the effect of SPC in the SGZ and GCL (Fig. 4C, D, HI + Sev + G versus $\mathrm{HI}+\mathrm{Sev}, \mathrm{p}<0.05, \mathrm{p}<0.05$ in SGZ; $<<0.01, \mathrm{p}<0.001$ in GCL on PND14 and PND21, respectively). 
Nissl staining was performed on PND14 to study the role of Reelin in the distribution of hippocampal DG neurons (Fig. 5A). The finding was consistent with the previous result that the HI group showed decreased neuron ratio with rare cytoplasm and few Nissl bodies, as well as disorganized neurons without polarity in DG as compared to that in the Sham group (Fig. $5 B, H$ versus Sham, $p<0.0001$ ). SPC alleviated neuron loss and promoted the reorganization of neurons with proper orientation (Fig. 5B, $\mathrm{HI}+$ Sev versus $\mathrm{HI}, \mathrm{p}<0.01$ ). The beneficial effect of SPC was, however, blocked by Piceatannol (Fig. 5B, $\mathrm{HI}+$ $\mathrm{Sev}+\mathrm{G}$ versus $\mathrm{HI}+\mathrm{Sev}, \mathrm{p}<0.05$ )

SPC attenuated DG neuron migration disorder in HIE by Reelin/Dab1 Cascade and inhibition of Reelin cleavage blocked this effect

The staining data indicate that HIE may result in disorder of neuron migration and activation of intracellular Dab1 expression by enhanced Reelin expression. Here, we tested the role of the canonical Reelin downstream signaling pathway involving Dab1, GSK-3ß, and the microtubule-associated protein Tau in HIE-induced neuron migration disorder. The HI group showed significant upregulation of Reelin expression in $388 \mathrm{kDa}$ fragment, rather than $180 \mathrm{kDa}$ and phosphorylation/activation of Dab1 (Tyr-198), GSK-3 $\beta$ (Ser9), and Tau (Ser396) as compared to that in the Sham group(Fig. 6A-D, HI versus Sham, $p<$ $0.001, p<0.01, p<0.01, p<0.05$, respectively). These reductions were significantly attenuated by SPC (Fig. 6A-D, HI +Sev versus $\mathrm{HI}, \mathrm{p}<0.001, \mathrm{p}<0.05, \mathrm{p}<0.05, \mathrm{p}<0.01$, respectively). Piceatannol partially blocked the effect of SPC (Fig. 6A-D, HI + Sev + G versus $H I+$ Sev, $p<0.05, p<0.01, p<0.05, p<0.05$, respectively).

SPC promotes hippocampal spatial learning and memory in HIE rats

To exclude anxiety and locomotor ability interference produced by several treatments, we conducted an open field test experiment on PND28. However, there were no difference in the test results among the four groups (Supplementary Figure S3).

In the MWM test, no significant difference in swimming velocities was observed among the four groups (Fig. 7A, $p>0.05$ among all groups). Rats in the $\mathrm{HI}$ group showed significantly increased escape latency as compared to rats in the Sham group (Fig. 7B, HI versus Sham, $p<0.001, p<0.001, p<0.001$, and $p<$ 0.01 on the $2 \mathrm{nd}, 3 \mathrm{rd}, 4$ th, and 5 th day, respectively). SPC attenuated this phenomenon (Fig. 7B, HI + Sev versus $\mathrm{HI}, \mathrm{p}<0.01, \mathrm{p}<0.001$, and $\mathrm{p}<0.01$ on the 3rd, 4th, and 5th day, respectively). Rats in the $\mathrm{HI}+\mathrm{Sev}+$ $G$ group treated with Piceatannol needed more time to find the platform than rats who were administered SPC alone (Fig. 7B, $\mathrm{HI}+\mathrm{Sev}+\mathrm{G}$ versus $\mathrm{HI}+\mathrm{Sev}, \mathrm{p}<0.01$ and $\mathrm{p}<0.05$ on the 4 th and 5 th day, respectively). In the spatial probe test, the $\mathrm{HI}$ group rats showed crossed the platform less number of times than the Sham group rats (Fig. 7C, HI versus Sham, $\mathrm{p}<0.01$ ). Rats in the HI+Sev group crossed the platform a larger number of times than rats in the $\mathrm{HI}$ group (Fig. $7 \mathrm{C}, \mathrm{HI}+\mathrm{Sev}$ versus $\mathrm{HI}, \mathrm{p}<0.05$ ). Piceatannol notably blocked the protective effect of SPC on spatial learning ability (Fig. $7 \mathrm{C}, \mathrm{HI}+\mathrm{Sev}+\mathrm{G}$ versus $\mathrm{HI}+\mathrm{Sev}, \mathrm{p}<$ 0.05).

SPC facilitates DG-dependent spatial learning and memory in HIE rats 
The 8-arm radial maze test was conducted in rats of the four groups from PND28 to PND34, and the path parameters were presented (Fig. 8A). The distance traveled during the reward seeking process was increased in the HI group, but significantly decreased in the $\mathrm{HI}+\mathrm{Sev}$ group. Rats in the HI + Sev + G group traveled more distance to find the rewards than rats in the $\mathrm{HI}+\mathrm{Sev}$ group (Fig. 8B, HI versus Sham, $\mathrm{p}<$ 0.001, $p<0.0001 ; H I+$ Sev versus $H I, p<0.05, p<0.05 ; H I+S e v+G$ versus $H I+S e v, p<0.05, p<0.05)$. SPC abolished the increased RME of the $\mathrm{HI}$ group as compared to that of the Sham group (Fig. $8 \mathrm{C}$, HI versus Sham, $\mathrm{p}<0.001 ; \mathrm{HI}+\mathrm{Sev}$ versus $\mathrm{HI}, \mathrm{p}<0.05)$, and Piceatannol blocked this beneficial effect (Fig. $8 \mathrm{C}, \mathrm{HI}+$ Sev $+G$ versus $H I+S e v, p<0.05)$. Interestingly, WME, which represents short-term memory, showed no difference among the four groups (Fig. 8D, p $>0.05$ compared with all groups). This indicated that the specific hippocampal region that controlled short-term memory production might not be influenced by HIE or SPC.

\section{Discussion}

Our study showed that HIE perturbed the neuron migration process and disordered DG neurons, leading to long-lasting neurological deficits. SPC can abolish these impairments and finally facilitate hippocampaldependent long-term spatial learning and cognition. The relative mechanism of SPC action may involve regulation of Reelin expression and suppression of the Reelin/Dab1/GSK-3ß/Tau signaling pathway to modulate the dynamics of cytoskeleton assembly in DG neurons.

$\mathrm{HIE}$ is a devastating consequence of perinatal asphyxia that leads to severe brain structure aberration and long-term neurocognitive sequela such as mental retardation ${ }^{22-25}$. The lack of an effective therapy for brain injury has therefore aroused intense interest in investigating appropriate drugs for administration. To our knowledge, sevoflurane is a popular general inhaled anesthetic that is particularly used in pediatric operation. In addition to its traditionally accepted protective effect by decreasing neuronal death and promoting long-term memory consolidation ${ }^{26}$, an appropriate dose of SPC may facilitate endogenous hippocampal neural network reconstruction through neurogenesis and promotion of migration of astrocytes and microglia to the infarcted region for neurocognitive repairment 27,28 . Moreover, Chai reported that multiple use and overdose of sevoflurane in the pregnant period may directly inhibit neural migration in the cortex of offspring ${ }^{29}$. In our present study, we first investigated DG neuron migration and neuron organization after HIE. Consistent with the findings of previous studies, we found that the normal DG neuron migration process was significantly perturbed and neural orientation was disordered after HIE. SPC administration functionally ameliorated these impairments.

DG of the hippocampus is special trilaminar, C-shaped structure where neurogenesis occurs postnatally and has a pivotal role in learning and memory ${ }^{30}$. Progenitors are generated in DG and migrate short distance from the inner SGZ to the superficial molecular layer; this process is mediated by other intercellular factors. Reelin, for example, integrates with the pre-existing hippocampal circuit and forms functional synaptic attachments almost in 1 week ${ }^{31,32}$. Here, we chose 7 -day-old rats as experimental candidates. This programmed process is, however, vulnerbable to hypoxia and changes in the 
extracelluar matrix, which result in hippocampal neuron migration deficits, leading to aberrations in the orchestration and dysfunction of the hippocampus ${ }^{24}$. The indicated mechanisms that modulate the migration of newborn neurons to their destination are more clear in human health studies, for example, disruptions of neuronal migration for cortex development may induce lissencephaly and seizure ${ }^{33}$. Consistent with other studies, our study data showed that Reelin expression was significantly enhanced after HIE. SPC normalized Reelin expression, which indicates that its neuroprotective effect on reconstructing DG structure may involve modulation of Reelin expression.

Reelin is a 388-kDa extracelluar matrix glycoprotein with several fragments and plays important roles in cortical and hippocampal lamination, synaptogenesis, neurite formation, and neurotransmitter release ${ }^{34}$, ${ }^{35}$. Furthermore, accumulating evidence showed that disrupted Reelin expression may be a potential reason for various neurodegenerative disorders such as seizure and mental retardation ${ }^{36,37}$. When the canonical Reelin-dependent Dab1 is phosphorylated, it activates downstream signaling cascades and acts as a stop signal in neuron migration ${ }^{38}$. A previous study also revealed that Reelin can stabilize actin cytoskeleton and microtubule cytoskeleton by binding to ApoER2 or VLDLR receptors to promote nuclear translocation acting on actin and microtubule cytoskeleton ${ }^{39}$. Evidence based on prenatal hypoxiainduced impaired neural migration emphasized the relationship between Reelin-associated migration deficits and hypoxia stimuli. 40 In addition, the reduced expression of Reelin during the prenatal period might lead to hippocampal-dependent cognitive impairments in adults ${ }^{41}$. It is reasonable to hypothesize that sevoflurane could ameliorate the effects of HIE by regulating DG neuron migration and neurological behaviors. Our study data revealed that SPC may facilitate the perturbed migration process of DG neurons in HIE by markedly disengaging perturbed prelabeled BrdU+/Neun + cells from the SGZ zone and promoting their percentage in the GCL from PND14 to PND21. Piceatannol, which directly increases Reelin level, blocked SPC function. This phenomenon implied the effect of Reelin on regulating DG lamination and Reelin/Dab1 interaction played the vital role in SPC-induced alleviation of DG developmental deficits after HIE.

The role of canonical Reelin-mediated signaling pathway in stabilizing the cytoskeleton by binding with receptors to activate Dab1-associated cascade has been highly debated ${ }^{42}$. This cytoskeleton rearrangement ability of modifying Tau and MAP1B might involve proline-directed kinase GSk3 and CDK5 43 . A study on the relationship between Reelin-mediated neuron migration process and microtubules showed that Reelin dynamically promoted the polymerization of microtubules, leading to the process of perturbed nuclear translocation ${ }^{44}$. In the present study, HIE obviously enrich Reelin expression and activated phopho-Dab1 at Tyr198, phosphor-GSK-3 $\beta$ at Ser 9 and phosphorylated Tau at Ser396 level. SPC can suppress the activated Reelin/Dab1/GSK-3ß/Tau signaling pathway. Notably, an injection of Piceatannol abolished this phenomenon, suggesting that Reelin/Dab1 pathway which influence microtubules polymerization did involve in the protection of SPC after HIE. This indicates that HIE may cause the spread of DG neurons without polarity, and this malformation may be associated with abnormal cleavage by Reelin and overactivation of the Reelin-Dab1 cascade. This damage may be reduced to some extent by SPC. 
To our knowledge, endogenous hippocampal events, including neurogenesis and neuron migration contribute to hippocampal memory and learning ${ }^{45-47}$. To verify whether SPC promotes long-term hippocampal memory after HIE, the MWM test was conducted. The results demonstrated that in the absence of motor disability and anxiety motion, hippocampal spatial and learning memory were extremely damaged by HIE. Gilber ${ }^{\mathrm{t} 48}$ showed that colchicine-induced DG lesion damaged DG-dependent learning ability. We conducted the 8-arm radial maze test to investigate DG-dependent pattern separation by referring to the capacity of transforming similar memories or events into nonoverlapping representations ${ }^{28}$. Our study data showed that RME, which represents long-term memory, was increased in the $\mathrm{HI}$ group. The phenomenon that rats in the $\mathrm{HI}$ group entered the working arm of the 8-arm maze without food rewards after training was abolished significantly by SPC. Piceatannol injection showed a similar harmful effect as that observed for the HI group. Interestingly, WME, which represents short-term memory retrieval, showed no difference among the four groups. This finding implied that HIE may particularly damage DG-dependent long-term memory storage instead of short-term memory, which suggests that HIE-triggered neuron migration disorder influenced the integration of short-term memory into long-term memory instead of inhibiting its production. These results are consistent with a previous research on traumatic brain injury ${ }^{49}$.

Our studied have several limitations. First, we mostly focused on clarifying the neuron migration disorder resulting from HIE and the neuroprotective effect of SPC on modulating this impairment, instead of neurogenesis and relative molecular mechanisms. Second, we used BrdU pulse-labeled technique, which may add difficulties in absolutely qualifying and tracing single neuron migration destination. We are currently working on our transgenic mice and a new method and may present the dynamics of the neuron migration pathway in a better way in further investigations.

\section{Declarations}

1. Ethics approval and consent to participate

All experimental procedures were approved by The Laboratory Animal Care Committee of China Medical University (Shenyang, China; Approval No.: 2016PS337K) and conformed to National Institutes of Health Guidelines for the Care and Use of Laboratory Animals.

\section{Consent for publication}

Not applicable

\section{Availability of data and materials}

The datasets used and/or analysed during the current study are available from the corresponding author on reasonable request.

\section{Funding}


This work was supported by grant the Natural Science Foundation of China (No. 81671311, 81870838), the Key Research and Development Program of Liaoning Province (No. 2018225004) and the Outstanding Scientific Fund of Shengjing Hospital (No. 201708)

\section{Competing interests}

The authors declare that they have no competing interests

\section{Authors' contributions}

$Y Z$ and $P Z$ contributions to research design. $Y Z, Q G$, and $P Z$ contributed to the planning of the work. $Y Z$ performed all the experiments with the help of QG, HX and ZW. YZ, QG, XH and CL participated in the analysis or interpretation of data. YZ wrote the manuscript. PZ approved of the submission and final versions.

\section{Acknowledgements}

Not applicable

\section{References}

1. Ma Q, Dasgupta C, Li Y, et al. MicroRNA-210 Downregulates ISCU and Induces Mitochondrial Dysfunction and Neuronal Death in Neonatal Hypoxic-Ischemic Brain Injury. Mol Neurobiol 2019. DOI: $10.1007 / \mathrm{s} 12035-019-1491-8$.

2. Zhao H, Mitchell S, Koumpa S, et al. Heme Oxygenase-1 Mediates Neuroprotection Conferred by Argon in Combination with Hypothermia in Neonatal Hypoxia-Ischemia Brain Injury. Anesthesiology 2016; 125: 180-192. DOI: 10.1097/ALN.0000000000001128.

3. Ming GL and Song H. Adult neurogenesis in the mammalian central nervous system. Annu Rev Neurosci 2005; 28: 223-250. DOI: 10.1146/annurev.neuro.28.051804.101459.

4. Piatti VC, Esposito MS and Schinder AF. The timing of neuronal development in adult hippocampal neurogenesis. Neuroscientist 2006; 12: 463-468. DOI: 10.1177/1073858406293538.

5. MA M, EH L and C I. The science of stroke: mechanisms in search of treatments. Neuron 2010; 67: 181-198. DOI: 10.1016/j.neuron.2010.07.002.

6. $Q Y, W Y, X L$, et al. Activation of canonical notch signaling pathway is involved in the ischemic tolerance induced by sevoflurane preconditioning in mice. Anesthesiology 2012; 117: 996-1005. DOI: 10.1097/ALN.0b013e31826cb469.

7. Xue H, Xu Y, Wang S, et al. Sevoflurane post-conditioning alleviates neonatal rat hypoxic-ischemic cerebral injury via Ezh2-regulated autophagy. Drug Des Devel Ther2019; 13: 1691-1706. DOI: 10.2147/DDDT.S197325.

8. Ren X, Wang Z, Ma H, et al. Sevoflurane postconditioning provides neuroprotection against brain hypoxia-ischemia in neonatal rats. Neurol Sci 2014; 35: 1401-1404. DOI: 10.1007/s10072-014-1726- 
4.

9. Wang S, Xue H, Xu Y, et al. Sevoflurane Postconditioning Inhibits Autophagy Through Activation of the Extracellular Signal-Regulated Kinase Cascade, Alleviating Hypoxic-Ischemic Brain Injury in Neonatal Rats. Neurochem Res 2018. DOI: 10.1007/s11064-018-2682-9.

10. L L, H S, J X, et al. Sevoflurane preconditioning induced endogenous neurogenesis against ischemic brain injury by promoting microglial activation. Oncotarget 2017; 8: 28544-28557. DOI: 10.18632/oncotarget.15325.

11. Ogden KK, Ozkan ED and Rumbaugh G. Prioritizing the development of mouse models for childhood brain disorders. Neuropharmacology 2016; 100: 2-16. DOI: 10.1016/j.neuropharm.2015.07.029.

12. Kohno S, Kohno T, Nakano Y, et al. Mechanism and significance of specific proteolytic cleavage of Reelin. Biochem Biophys Res Commun 2009; 380: 93-97. DOI: 10.1016/j.bbrc.2009.01.039.

13. Kubo K, Mikoshiba K and Nakajima K. Secreted Reelin molecules form homodimers. Neurosci Res 2002; 43: 381-388.

14. G DA. The reeler mouse: anatomy of a mutant. International review of neurobiology 2005; 71 : 383417. DOI: 10.1016/s0074-7742(05)71016-3.

15. $A D L, M H O$ and AJ K. Extracellular matrix control of dendritic spine and synapse structure and plasticity in adulthood. Frontiers in neuroanatomy 2014; 8: 116. DOI: 10.3389/fnana.2014.00116.

16. Golan MH, Mane R, Molczadzki G, et al. Impaired migration signaling in the hippocampus following prenatal hypoxia. Neuropharmacology 2009; 57: 511-522. DOI: 10.1016/j.neuropharm.2009.07.028.

17. Komitova M, Xenos D, Salmaso N, et al. Hypoxia-induced developmental delays of inhibitory interneurons are reversed by environmental enrichment in the postnatal mouse forebrain. $J$ Neurosci 2013; 33: 13375-13387. DOI: 10.1523/JNEUROSCI.5286-12.2013.

18. Xu Y, Tian Y, Tian Y, et al. Autophagy activation involved in hypoxic-ischemic brain injury induces cognitive and memory impairment in neonatal rats. J Neurochem 2016; 139: 795-805. DOI: 10.1111/jnc. 13851.

19. Bingham B, Liu D, Wood A, et al. Ischemia-stimulated neurogenesis is regulated by proliferation, migration, differentiation and caspase activation of hippocampal precursor cells. Brain Res 2005; 1058: 167-177. DOI: 10.1016/j.brainres.2005.07.075.

20. Bartley J, Soltau T, Wimborne $\mathrm{H}$, et al. BrdU-positive cells in the neonatal mouse hippocampus following hypoxic-ischemic brain injury. BMC Neurosci 2005; 6: 15. DOI: 10.1186/1471-2202-6-15.

21. Pan YW, Chan GC, Kuo CT, et al. Inhibition of adult neurogenesis by inducible and targeted deletion of ERK5 mitogen-activated protein kinase specifically in adult neurogenic regions impairs contextual fear extinction and remote fear memory. J Neurosci 2012; 32: 6444-6455. DOI: 10.1523/JNEUROSCI.6076-11.2012.

22. Barkhuizen $M$, van den Hove DL, Vles JS, et al. 25 years of research on global asphyxia in the immature rat brain. Neurosci Biobehav Rev 2017; 75: 166-182. DOI:

10.1016/j.neubiorev.2017.01.042. 
23. Li Y, Gonzalez P and Zhang L. Fetal stress and programming of hypoxic/ischemic-sensitive phenotype in the neonatal brain: mechanisms and possible interventions. Prog Neurobiol 2012; 98 : 145-165. DOI: 10.1016/j.pneurobio.2012.05.010.

24. Ma Q and Zhang L. Epigenetic programming of hypoxic-ischemic encephalopathy in response to fetal hypoxia. Prog Neurobiol 2015; 124: 28-48. DOI: 10.1016/j.pneurobio.2014.11.001.

25. Millar LJ, Shi L, Hoerder-Suabedissen A, et al. Neonatal Hypoxia Ischaemia: Mechanisms, Models, and Therapeutic Challenges. Front Cell Neurosci 2017; 11: 78. DOI: 10.3389/fncel.2017.00078.

26. Wang Z, Ye Z, Huang G, et al. Sevoflurane Post-conditioning Enhanced Hippocampal Neuron Resistance to Global Cerebral Ischemia Induced by Cardiac Arrest in Rats through PI3K/Akt Survival Pathway. Front Cell Neurosci 2016; 10: 271. DOI: 10.3389/fncel.2016.00271.

27. Yu Q, Li L and Liang WM. Effect of sevoflurane preconditioning on astrocytic dynamics and neural network formation after cerebral ischemia and reperfusion in rats. Neural Regen Res 2019; 14: 265271. DOI: $10.4103 / 1673-5374.244790$.

28. Chen C, Shen FY, Zhao X, et al. Low-dose sevoflurane promotes hippocampal neurogenesis and facilitates the development of dentate gyrus-dependent learning in neonatal rats. ASN Neuro 2015; 7. DOI: $10.1177 / 1759091415575845$.

29. D C, Y C, Y S, et al. Multiple sevoflurane exposures during pregnancy inhibit neuronal migration by upregulating prostaglandin D2 synthase. International journal of developmental neuroscience : the official journal of the International Society for Developmental Neuroscience 2019; 78: 77-82. DOI: 10.1016/j.ijdevneu.2019.09.001.

30. Hevner RF. Evolution of the mammalian dentate gyrus. J Comp Neurol 2016; 524: 578-594. DOI: 10.1002/cne.23851.

31. Bruel-Jungerman E, Laroche $S$ and Rampon $C$. New neurons in the dentate gyrus are involved in the expression of enhanced long-term memory following environmental enrichment. Eur J Neurosci 2005; 21: 513-521. DOI: 10.1111/j.1460-9568.2005.03875.x.

32. Hack I, Bancila M, Loulier $\mathrm{K}$, et al. Reelin is a detachment signal in tangential chain-migration during postnatal neurogenesis. Nat Neurosci 2002; 5: 939-945. DOI: 10.1038/nn923.

33. G K and JG G. Genetic mechanisms underlying abnormal neuronal migration in classical lissencephaly. Trends in genetics : TIG 2007; 23: 623-630. DOI: 10.1016/j.tig.2007.09.003.

34. Nichols AJ and Olson EC. Reelin promotes neuronal orientation and dendritogenesis during preplate splitting. Cereb Cortex 2010; 20: 2213-2223. DOI: 10.1093/cercor/bhp303.

35. Levy AD, Omar MH and Koleske AJ. Extracellular matrix control of dendritic spine and synapse structure and plasticity in adulthood. Front Neuroanat 2014; 8: 116. DOI: 10.3389/fnana.2014.00116.

36. Pujadas L, Gruart A, Bosch C, et al. Reelin regulates postnatal neurogenesis and enhances spine hypertrophy and long-term potentiation. J Neurosci 2010; 30: 4636-4649. DOI: 10.1523/JNEUROSCI.5284-09.2010.

37. De Rubeis $\mathrm{S}, \mathrm{He} X$, Goldberg AP, et al. Synaptic, transcriptional and chromatin genes disrupted in autism. Nature 2014; 515: 209-215. DOI: 10.1038/nature13772. 
38. Bock HH and Herz J. Reelin activates SRC family tyrosine kinases in neurons. Curr Bio/ 2003; 13: 1826.

39. X C and M F. How does Reelin signaling regulate the neuronal cytoskeleton during migration? Neurogenesis (Austin, Tex) 2016; 3: e1242455. DOI: 10.1080/23262133.2016.1242455.

40. Frotscher $\mathrm{M}$, Haas $\mathrm{CA}$ and Forster $\mathrm{E}$. Reelin controls granule cell migration in the dentate gyrus by acting on the radial glial scaffold. Cereb Cortex 2003; 13: 634-640.

41. Pocock $R$ and Hobert 0 . Oxygen levels affect axon guidance and neuronal migration in Caenorhabditis elegans. Nat Neurosci 2008; 11: 894-900. DOI: 10.1038/nn.2152.

42. Chai $X$, Forster $E, Z$ hao $S$, et al. Reelin acts as a stop signal for radially migrating neurons by inducing phosphorylation of n-cofilin at the leading edge. Commun Integr Bio/ 2009; 2: 375-377.

43. Gonzalez-Billault C, Del Rio JA, Urena JM, et al. A role of MAP1B in Reelin-dependent neuronal migration. Cereb Cortex 2005; 15: 1134-1145. DOI: 10.1093/cercor/bhh213.

44. He M, Zhang ZH, Guan CB, et al. Leading tip drives soma translocation via forward F-actin flow during neuronal migration. J Neurosci 2010; 30: 10885-10898. DOI: 10.1523/JNEUROSCI.024010.2010 .

45. DN A and JM W. Interaction between Neurogenesis and Hippocampal Memory System: New Vistas. Cold Spring Harbor perspectives in biology 2015; 7. DOI: 10.1101/cshperspect.a018952.

46. G L, A N and PM L. Adult neurogenesis and the future of the rejuvenating brain circuits. Neuron 2015; 86: 387-401. DOI: 10.1016/j.neuron.2015.01.002.

47. M G, MF A, C M, et al. Hypoxic preconditioning confers long-term reduction of brain injury and improvement of neurological ability in immature rats. Pediatric research 2005; 57: 305-309. DOI: 10.1203/01.pdr.0000151122.58665.70.

48. Gilbert PE, Kesner RP and Lee I. Dissociating hippocampal subregions: double dissociation between dentate gyrus and CA1. Hippocampus 2001; 11: 626-636. DOI: 10.1002/hipo.1077.

49. Sebastian V, Diallo A, Ling DS, et al. Robust training attenuates TBI-induced deficits in reference and working memory on the radial 8-arm maze. Front Behav Neurosci 2013; 7: 38. DOI:

10.3389/fnbeh.2013.00038.

\section{Figures}




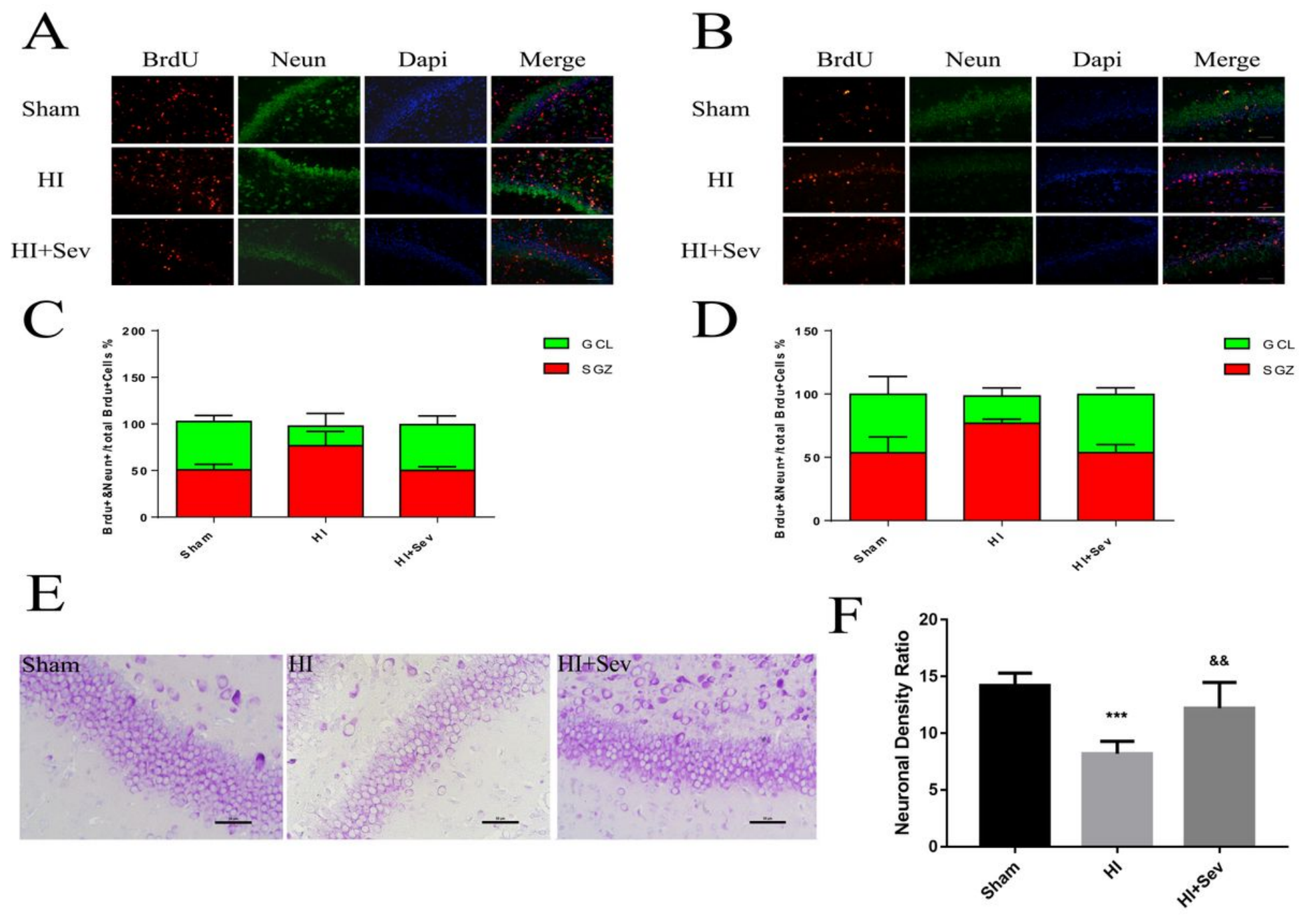

Figure 1

Perturbed Neuron migration process and disarrayed distribution of Dentate gyrus neuron HIE and effect of SPC on these deficits on PND14 and PND21. BrdU(red)/Neun(green)double-positive cell distributed in the SGZ zone(A) and GCL zone(B) onPND14 andPND21 respectively. The ratio of BrdU -positive neurons in the SGZ zone or GCL zone to BrdU-positive cells in the whole layer on PND14 (C),or PND21 (D)were calculated separately. Values are mean $\pm S D(n=3)$ two-way analysis of variance for repeated measurements followed by Tukey post-test and Mann-Whitney $U$ te was used for data analysis. Scale bar $=50 \mu \mathrm{m}$. Nissl staining was performed on PND14. Representative photographs of Nissl stained-Dentate gyrus $(E)$. Neuronal density relative ratio( $F)$ Values are mean $\pm \operatorname{SD}(n=5)$ One-way ANOVA with Tukey post hoc multiple comparison tests was used for data analysis. Scale bar $=50 \mu \mathrm{m} * \star \star p ~<0.001$ vs Sham group, \&\&p<0.01 vs $\mathrm{HI}+\mathrm{Sev}$ group. 
A

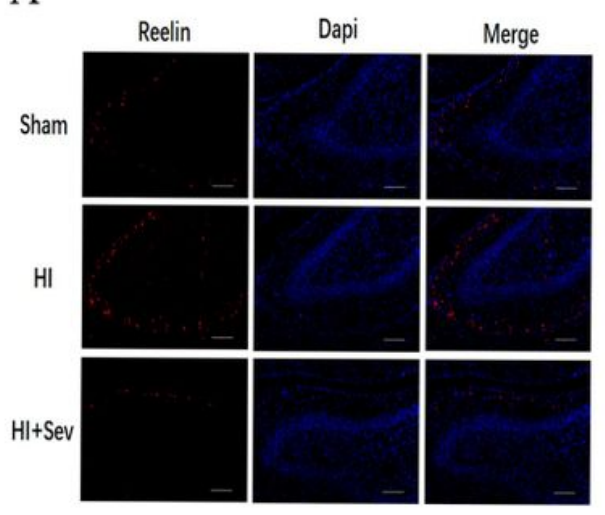

D

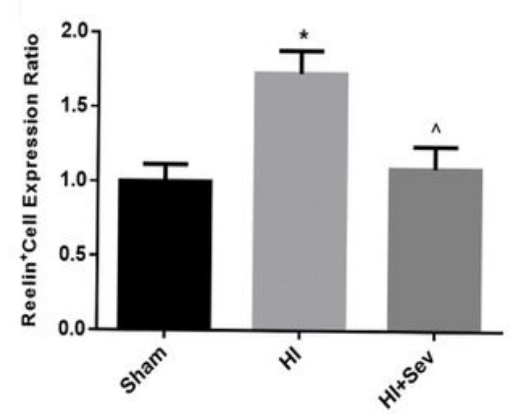

B

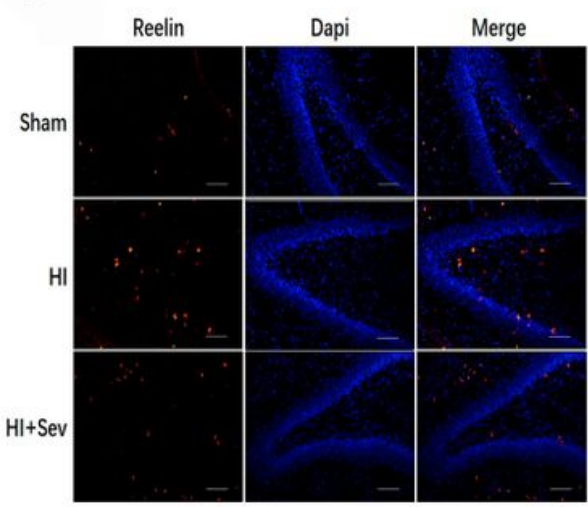

E

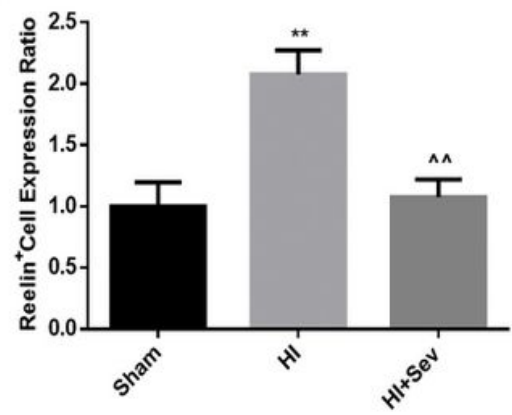

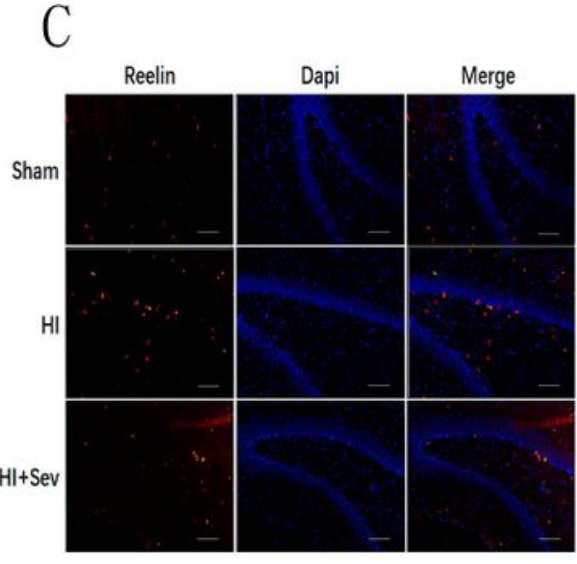

F

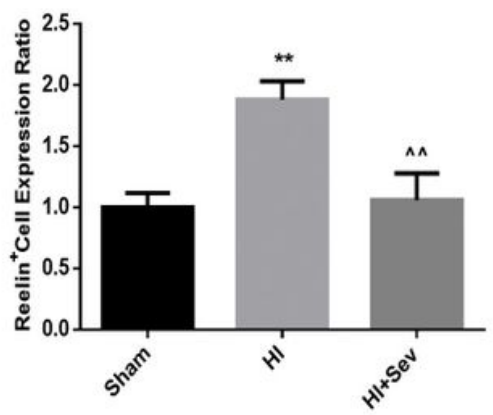

\section{Figure 2}

Reelin expression in dentate gyrus of hippocampus. (A-C) Immunofluorescence of Reelin(red) expression on PND8,14,21.(D-F) Quantitative analysis of RATIO OF reelin expression number in dentate gyrus on PND8(D) PND14(E)and PND21(F). Values are mean $\pm S D(n=5)$ One -way ANOVA with Tukey post hoc multiple comparison tests were used for data analysis. ${ }^{\star} p<0.05$ vs Sham group, \&\&p<0.001 vs HI group. 


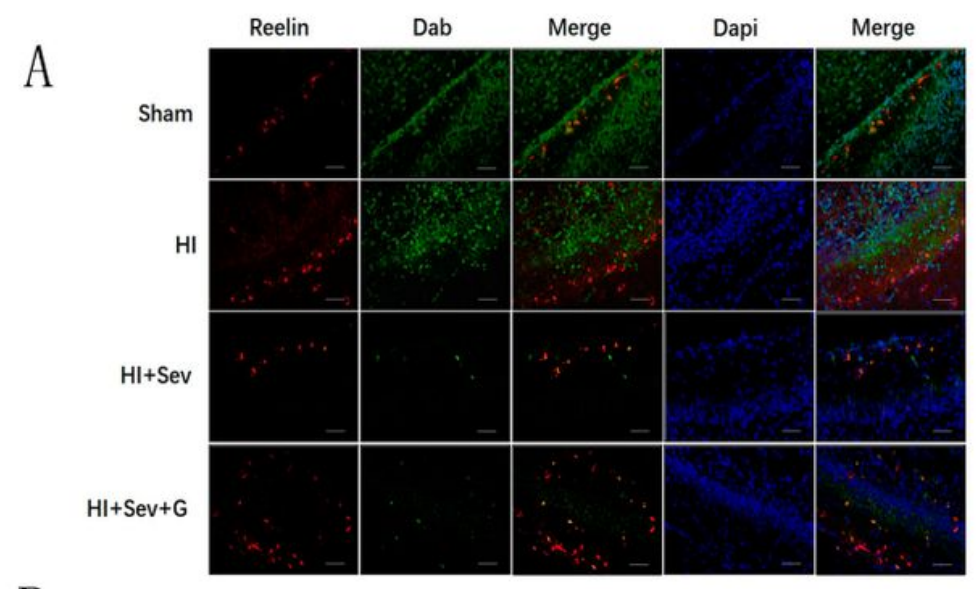

B
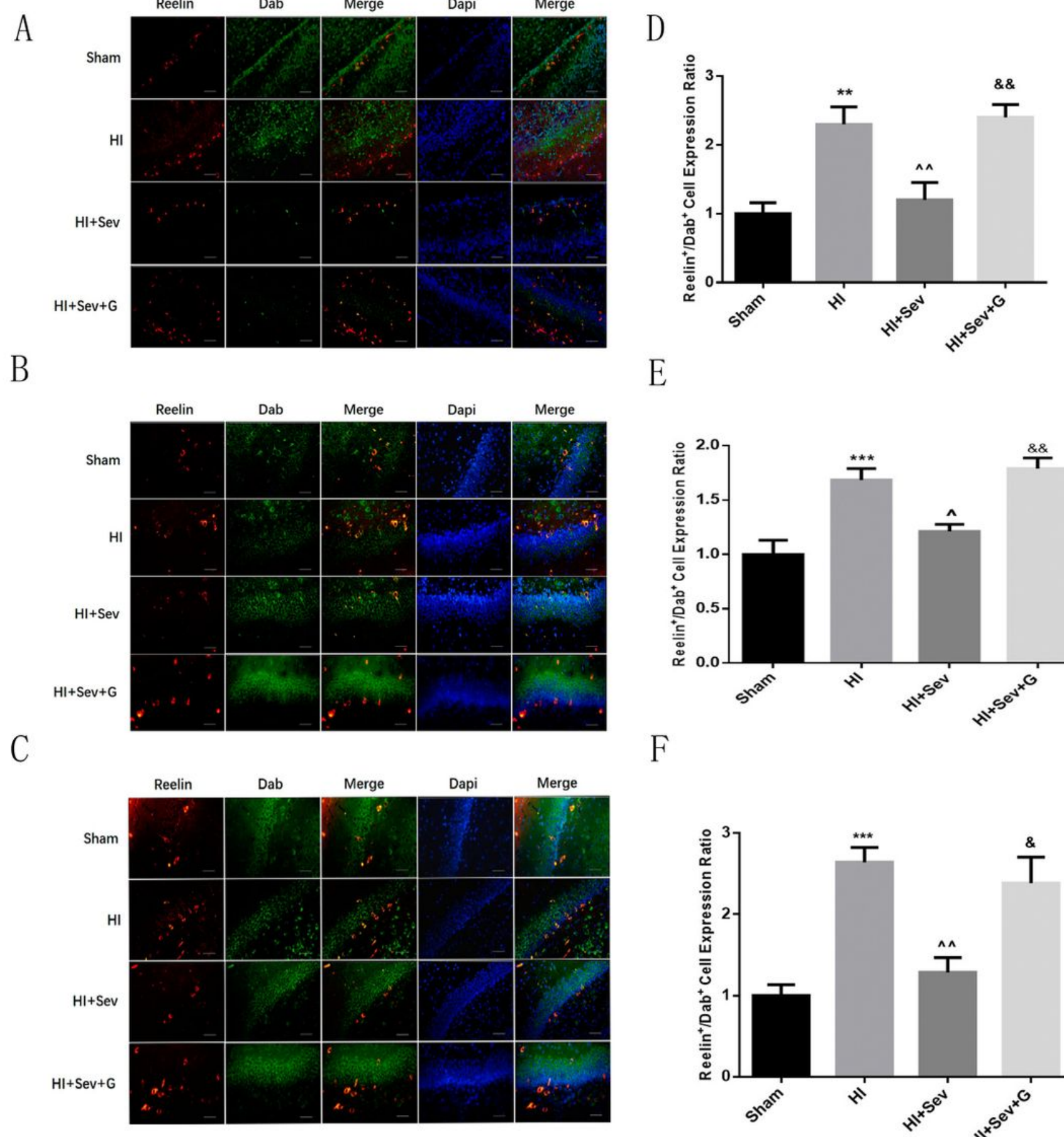

E
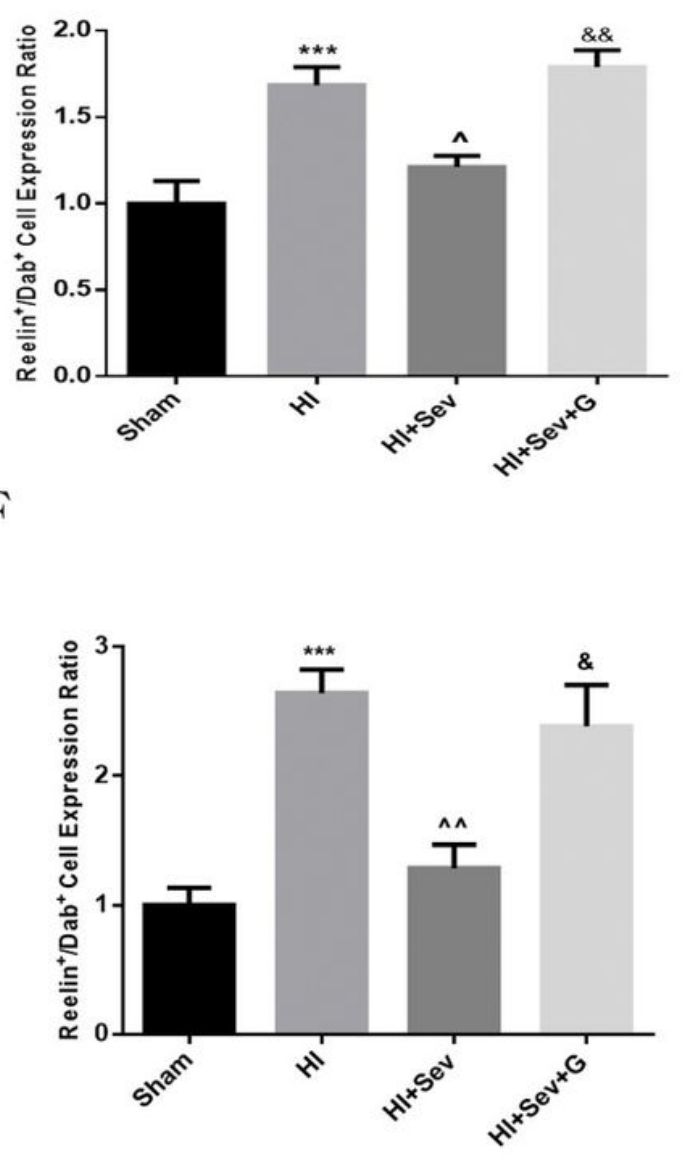

\section{Figure 3}

Reelin co-expression with Dab1 in dentate gyrus of hippocampus. Immunofluorescence of Reelin(red) colocalized with Dab1(green) on PND14(A)and PND21(B). Quantitative analysis of Reelin-Dab1 expression cell ratio on PND14(C) and PND21(D). Values are mean $\pm S D(n=5)$ One-way ANOVA with Tukey post hoc multiple comparison tests were used for data analysis. Scale bar $=50 \mu \mathrm{m}$. ${ }^{\star \star} p<0.01$, ${ }^{\star \star} \mathrm{p}$ $<0.001$ vs Sham group; ${ }^{\wedge} p<0.05,{ }^{\wedge \wedge} p<0.01$ vs HI group; \&p<0.05, \&\&p<0.01vs HI+Sev group. 

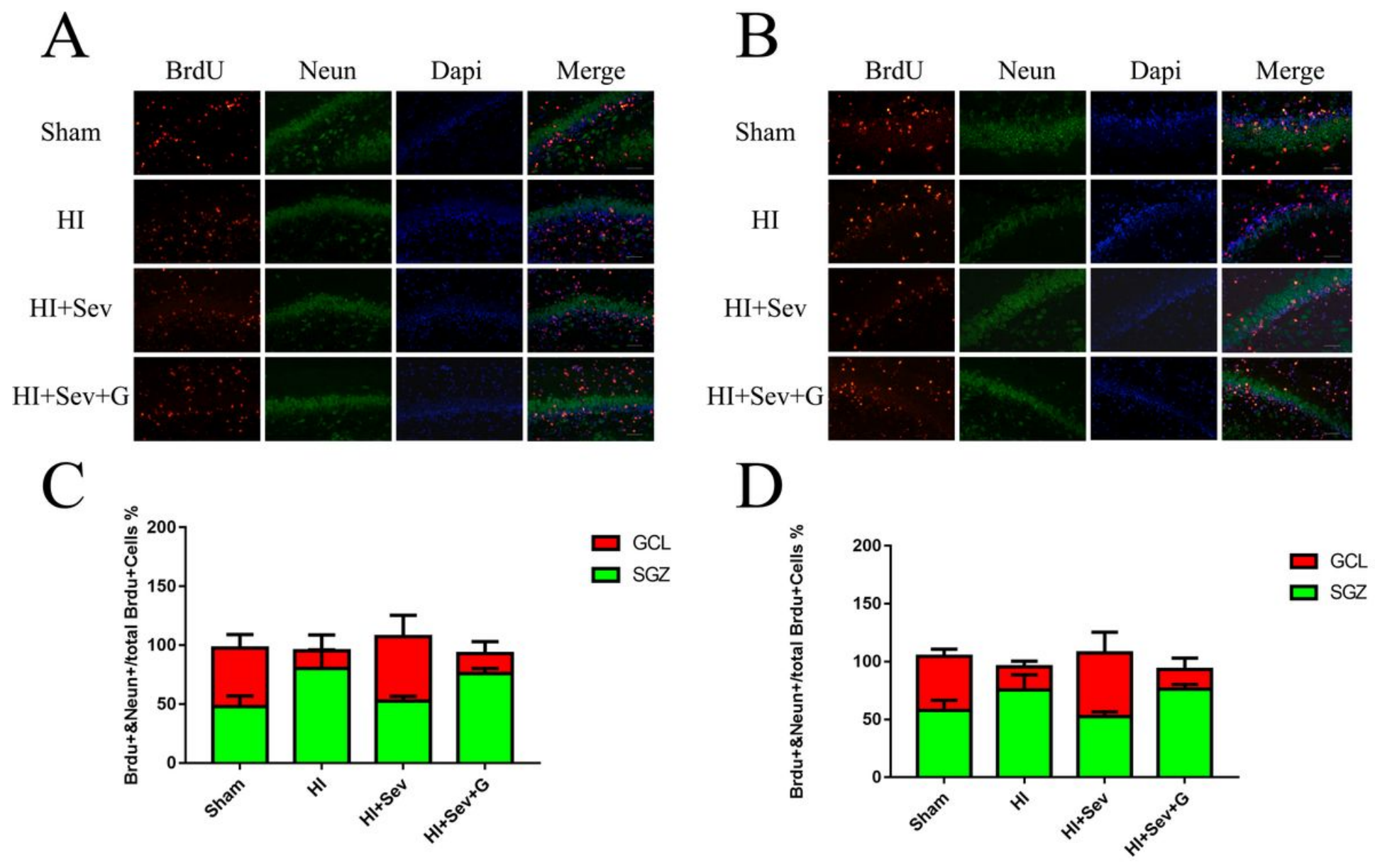

\section{Figure 4}

Immunofluorescence of neuron migration after HIE and SPC protective effect on it.

BrdU(red)/Neun(green)double-positive cell distributed in the SGZ zone(A) and GCL zone(B) onPND14 andPND21 respectively. The ratio of BrdU -positive neurons in the SGZ zone or GCL zone to BrdU-positive cells in the whole layer on PND14 (C),or PND21 (D)were calculated separately. Values are mean \pm $\mathrm{SD}(\mathrm{n}=3)$ two-way analysis of variance for repeated measurements followed by Tukey post-test and Mann-Whitney $U$ te was used for data analysis. Scale bar $=50 \mu \mathrm{m}$. 
A
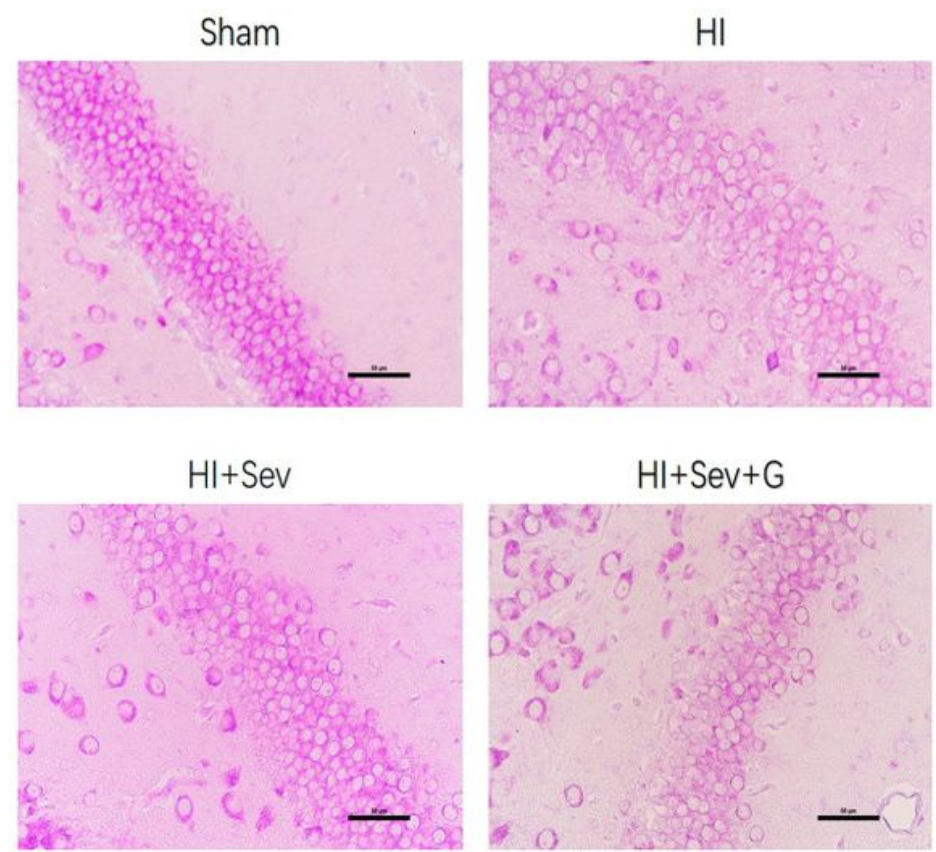

B

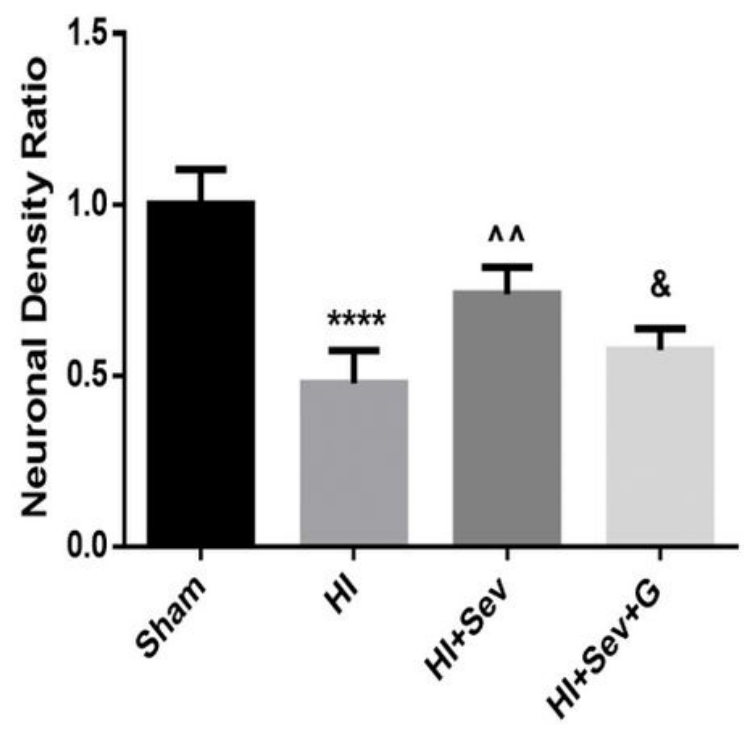

\section{Figure 5}

Nissl staining was performed on PND14. Representative photographs of Nissl stained-Dentate gyrus(E). Neuronal density relative ratio( $F)$ Values are mean $\pm S D(n=5)$ One-way ANOVA with Tukey post hoc multiple comparison tests was used for data analysis. Scale bar $=50 \mu m .{ }^{\star \star *} p<0.0001$ vs Sham group, ${ }^{\wedge} \mathrm{p}<0.01$ vs HI group, \&p<0.05vs HI+Sev group. 
A

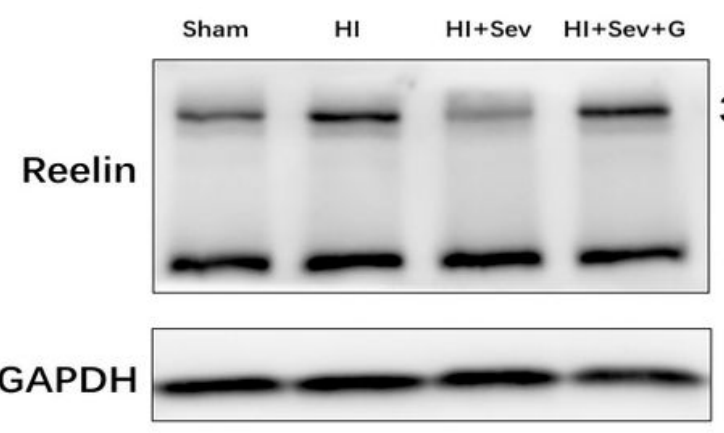

388kDa

$180 \mathrm{kDa}$

$36 \mathrm{kDa}$

C

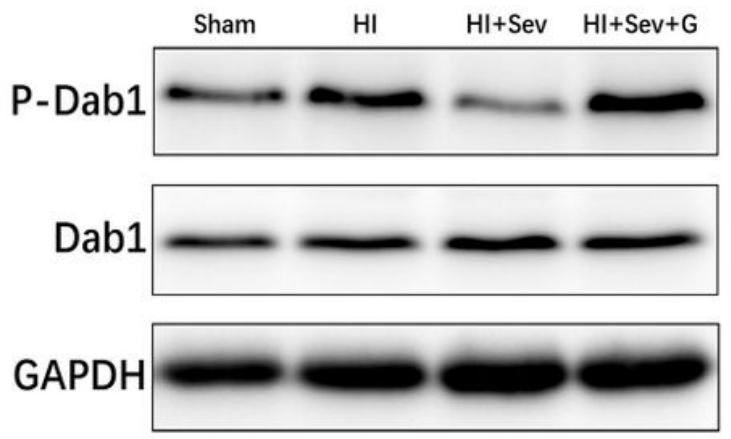

$60 \mathrm{kDa}$

$60 \mathrm{kDa}$

$36 \mathrm{kDa}$

E

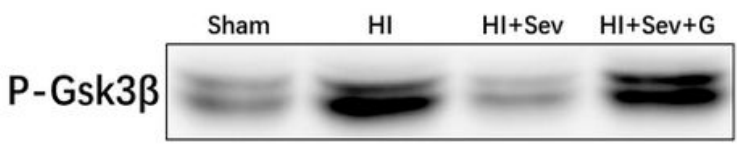

$46 \mathrm{kDa}$

Gsk3ß

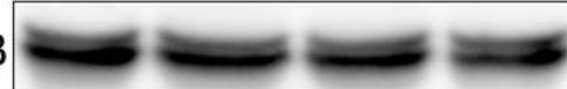

$46 \mathrm{kDa}$

GAPDH

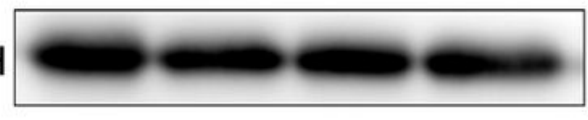

$36 \mathrm{kDa}$

G

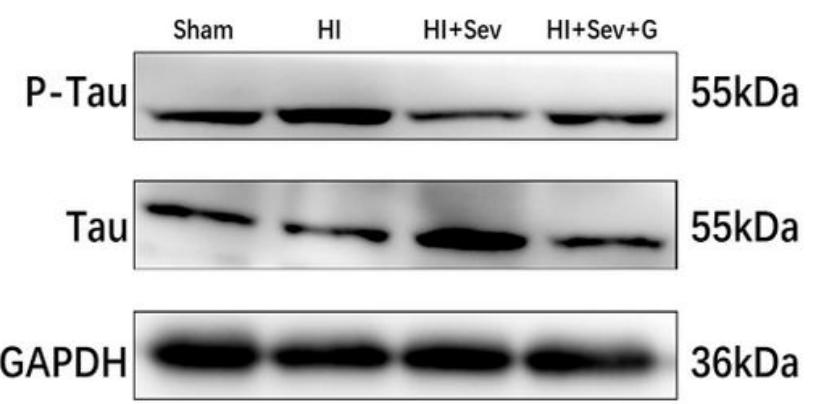

B

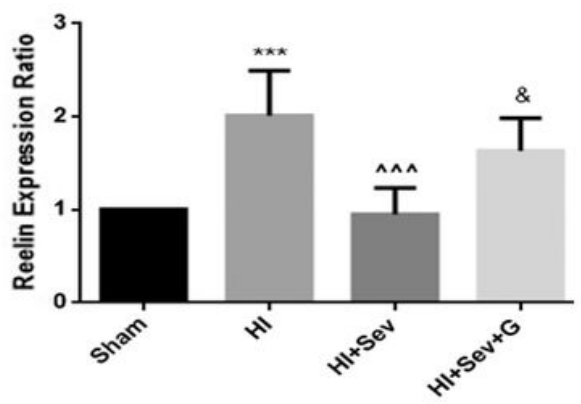

D

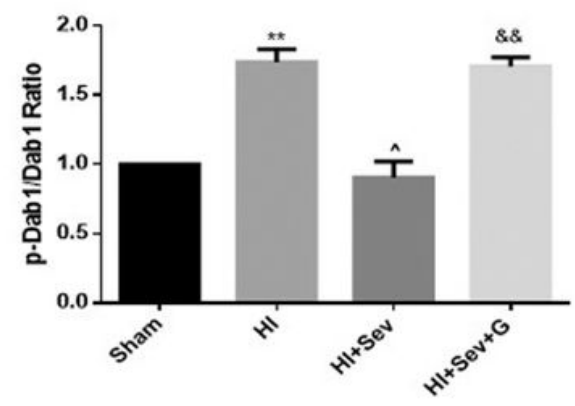

F

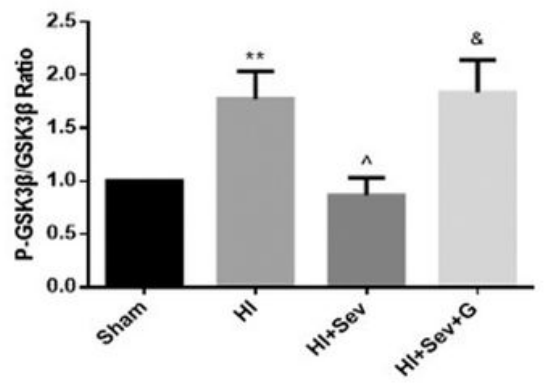

H

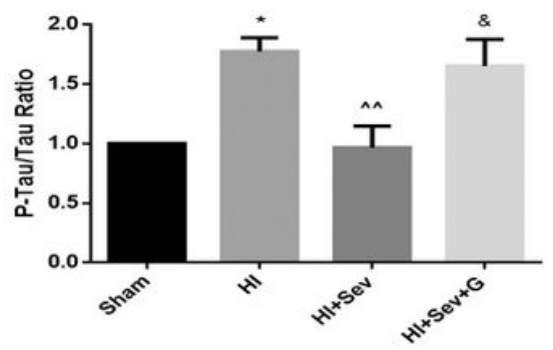

\section{Figure 6}

Sevoflurane Postconditioning Attenuated DG Migrating Aberration in HIE via Reelin/Dab1 Cascade. Target proteins were tested on PND8. Representative western blotting photographs(A,C,E,G). Quantitative analysis of Reelin expression level of 388kDa fragment(B). Quantitative analysis of P-Dab1/Dab1 level(D). Quantitative analysis of P-GSK-3/ GSK-3ß level(F). Quantitative analysis of P-Tau/Tau level (H). Values are mean \pm S.E.M. $(n=5)$. One-way ANOVA with Newman-Keulspost hoc test or Kruskal-Wallis 
with Dunn's Multiple comparison test was used for data analysis. ${ }^{*} p<0.05,{ }^{*} p<0.01 * \star * p<0.001$ vs Sham group; ${ }^{\wedge} p<0.05,{ }^{\wedge \wedge} p<0.01,{ }^{\wedge \wedge \wedge} p<0.001$ vs HI group; \&p<0.05,\&\&p<0.01 vs HI+Sev group.

A

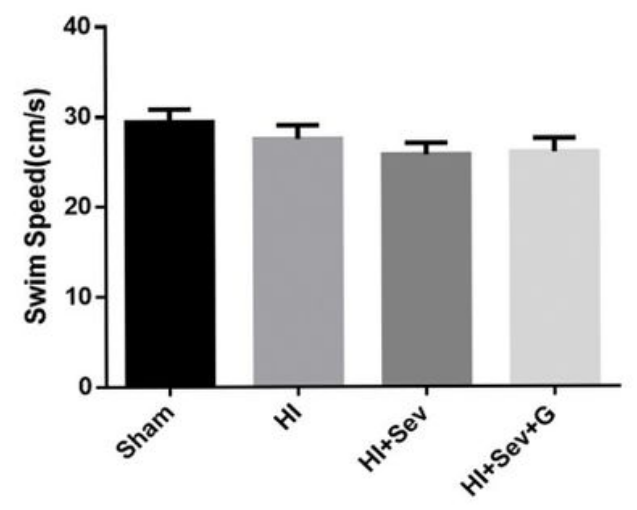

C

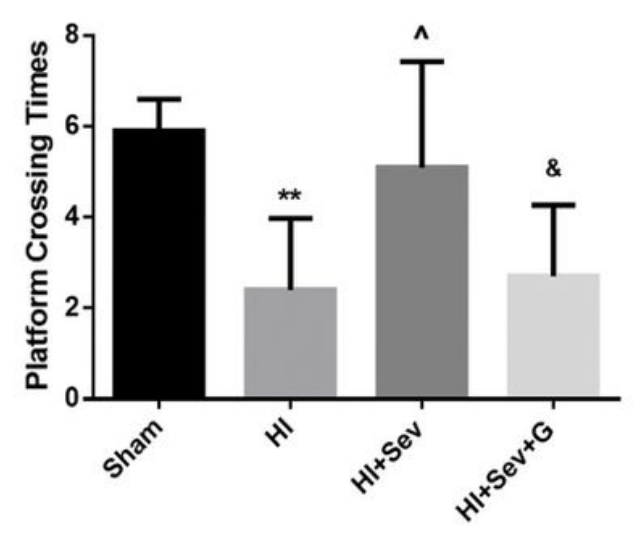

B

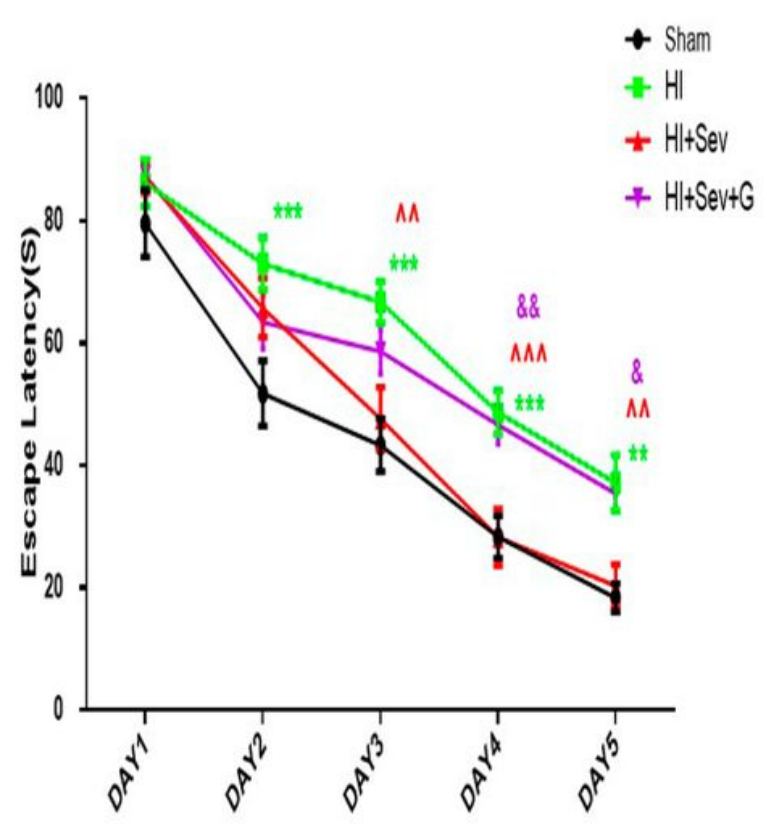

\section{Figure 7}

Morris water maze tests of the spatial learning and memory function. (A) The average swimming speed(A), Escape latency(B) and Platform crossing times (C) were tested in different groups. Values are mean \pm S.E.M. $(n=10)$ Escape latency calculated using two-way analysis of variance for repeated measurements followed by Tukey post-test and Mann-Whitney $U$ test was used to test the difference of 
platform crossing times and swimming velocity. ${ }^{\star \star \star} p<0.001$ vs Sham group, ${ }^{\wedge \wedge} p<0.01,{ }^{\wedge \wedge} p<0.001$ vs HI group; \&p<0.05, \&\&p<0.01vs HI+Sev group.

A

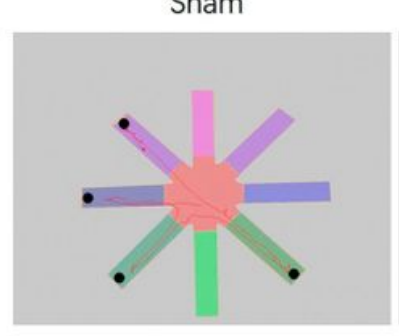

$\mathrm{HI}+\mathrm{SeV}$

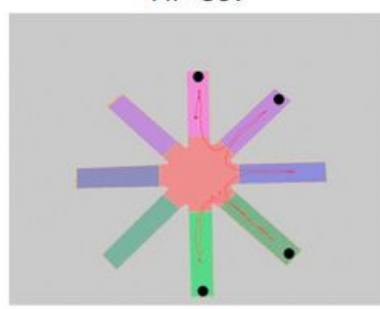

C

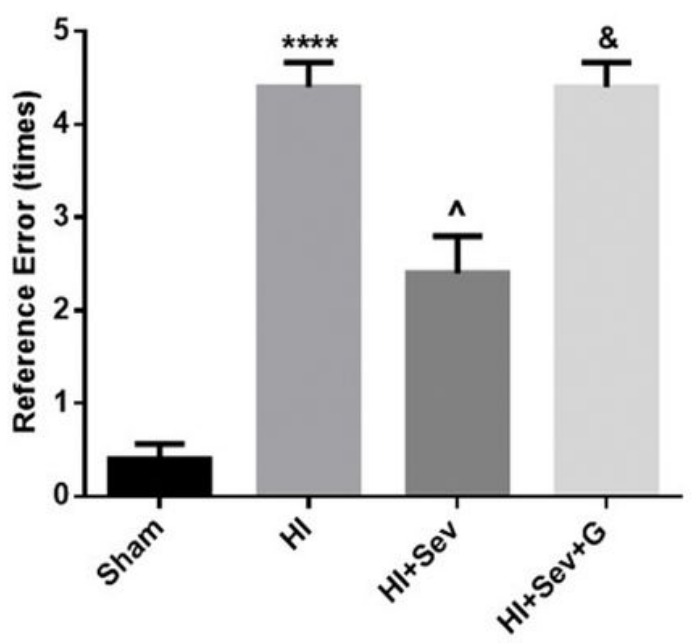

B
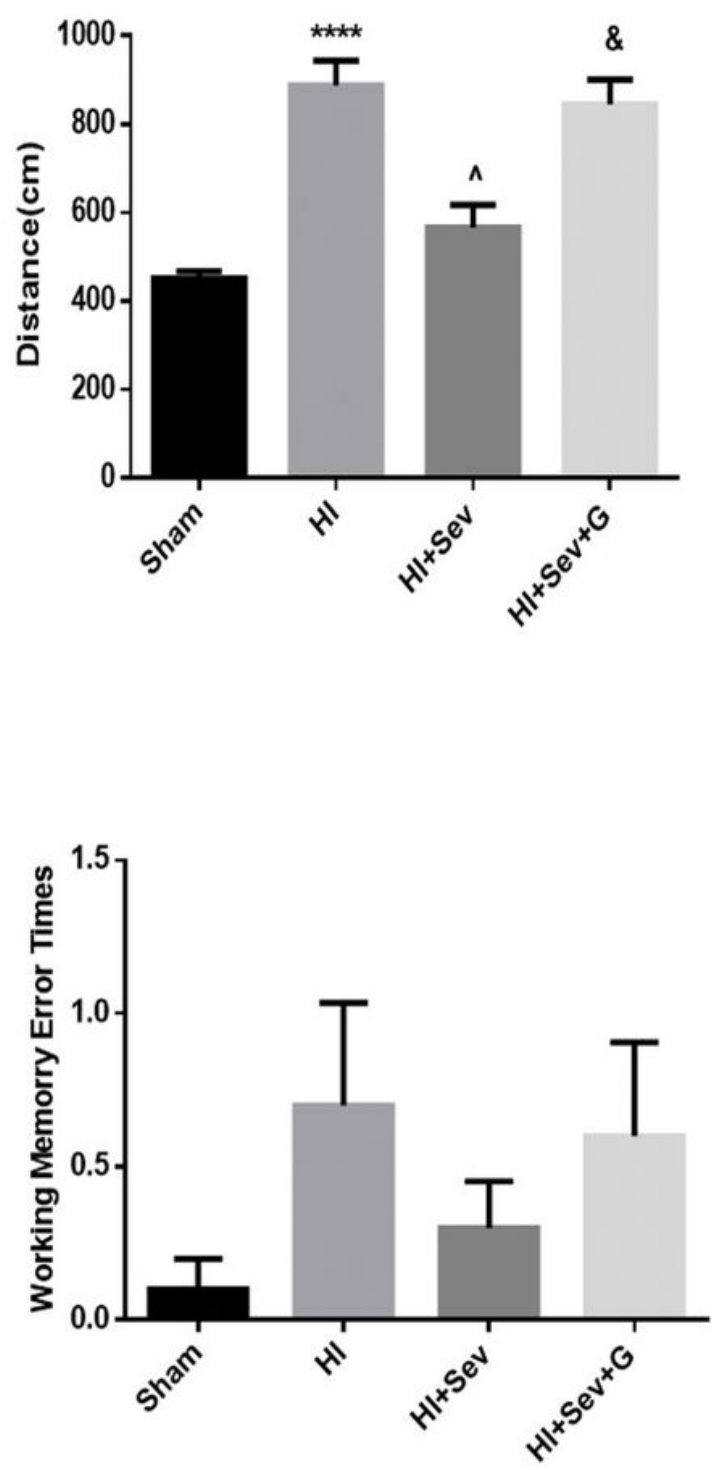

\section{Figure 8}

8-Arm-Maze tests of the dentate gyrus-dependent learning and memory function. Movement track(A)Movement distance of rats(B). Reference Memory Errors(C), Working Memory Errors(D). Values are mean \pm S.E.M $(n=10)$. One-way ANOVA with Newman-Keulspost hoc test or Kruskal-Wallis with 
Dunn's Multiple comparison test was used for data analysis. ${ }^{\star \star \star \star} p<0.0001$ vs Sham group; ${ }^{\wedge} p<0.05$ vs HI group; \&p<0.05 vs HI+Sev group.

\section{Supplementary Files}

This is a list of supplementary files associated with this preprint. Click to download.

- s1.jpg

- s3.tif

- S2.jpg 